\title{
Simulation Research on Logic Rule Control of Vehicle AFS
}

\author{
Wu Dirui \\ Wuhan University of Technology, School of Automotive Engineering, 430070 \\ Email: wudirui@outlook.com
}

Keywords: Adaptive Front-lighting System; mathematical model; logic rule control; simulation research

\begin{abstract}
Adaptive Front-lighting System (AFS) can adjust front light angle in real time based on speed, steering wheel angle and dip angle of vehicle. The safety of night driving can be improved in the active safety technology. Based on the mathematical model of AFS system, logic rule control method is adopted in this paper. The logic rule control system of automobile AFS is established. The simulation control model of AFS system in Matlab/Simulink is established, and the simulation of the model is carried out. The simulation results show that the system control parameters are easier to adjust and control the results have better dynamic and static performance.
\end{abstract}

\section{Introduction}

AFS (Front-lighting System Adaptive) is the full name of the adaptive front lighting system. It is the headlight axis in the horizontal direction and the steering wheel angle linkage to rotate left and right, and in the vertical direction and the vehicle high linkage swing light servo system. Statistics show that: in the night the traffic volume is about $80 \%$ lower than during the day, however, in the night leading to the death of traffic accidents accounted for more than $40 \%$ of all traffic accidents[1]. The AFS lighting system can significantly improve the safety of night driving, and greatly improves the comfort of driving lighting. It makes dipped beam of light automatically turn the forward direction of the vehicle, so it can improve the degree of fatigue in night driving visibility on the front of the intersection and effectively reduce the driver driving in the night detour [2].

The hardware system of the AFS system includes the body sensor, the AFS controller (ECU) and the executing units. AFS system controller collects steering wheel angle signal, vehicle speed and vehicle high signal. It can calculate and judge according to comprehensive factors about the dynamic changes of vehicle body, movement characteristics of steering mechanism and working status of engine, then obtained corresponding horizontal and vertical angle through the mathematical model. Thus the current running status of automobile are judged and the headlight near light can be adjusted correspondingly. AFS system is finally driven by the driver of two stepper motors to adjust the horizontal and vertical direction, to achieve the best lighting effect. The dynamic model of the AFS system explains the theory of the relationship between the driving information and the turning angle. But in practice, if the output result of the AFS dynamic model is directly dependent on the model to control the execution mechanism of the AFS system, this kind of controlled object with the characteristics of pure delay and large inertia has some problems, including existing some angle control error, not solving the system performance, difficulties to unify the stability, accuracy and speed, and the object parameters are sensitive to parameters, and the system robustness is not strong.

In view of the above problems, the logic rule control method is adopted in this paper, and the simulation control model is established in Matlab/Simulink, and the simulation research of AFS logic rule control system is carried on. 


\section{AFS logic rule control system and AFS system model}

\subsection{The logic rule controller}

Logic controller is according to the measured relationship between angle error and angle error change to judge the future response trend, logic controller makes the controlled object's output reach the set value in time. The basis of the control strategy is based on relationship between system error and error change The control structure diagram is shown in Fig.1. $\pm e_{0}$ are system error lower limit and upper limit respectively, $\pm \dot{e}_{0}$ are system error change lower limit and upper limit in Fig.1 [3].

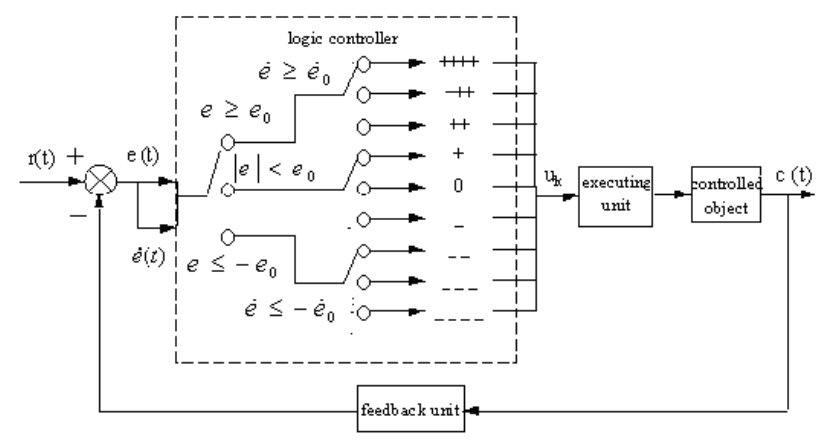

Fig. 1 The control structure diagram

\subsection{Formation of logic control strategy}

If in phase plane according to the relationship between the headlamp in horizontal and vertical direction angle error and error change, the phase plane is divided into nine working area, there will be nine operating conditions while the system is running, which correspond to nine kinds of control strategies. It can be represented by the logic control rule table 1[4]:

Table 1 The logic control rule table

\begin{tabular}{|c|c|c|c|}
\hline & $\dot{e} \geq \dot{e}_{0}$ & $|\dot{e}|<\dot{e}_{0}$ & $\dot{e} \leq-\dot{e}_{0}$ \\
\hline$e \geq e_{0}$ & more plus $k_{4}^{+}$ & plus $k_{3}^{+}$ & a little plus $k_{2}^{+}$ \\
\hline$|e|<e_{0}$ & slightly plus $k_{1}^{+}$ & keep invariant $k_{0}$ & slightly decrease $k_{1}^{-}$ \\
\hline$e \leq-e_{0}$ & slightly decrease $k_{2}^{-}$ & decrease $k_{3}^{-}$ & more decrease $k_{4}^{-}$ \\
\hline
\end{tabular}

In table1, nine control strategies are formed by the combination of $e$ and $\dot{e}$, a total of nine control strategies are adopted. $k_{4}^{+}, k_{3}^{+}, k_{2}^{+}, k_{1}^{+}, k_{0}, k_{1}^{-}, k_{2}^{-}, k_{3}^{-}, k_{4}^{-}$denote the adjustment angle value of the stepper motors more plus, plus, a little plus, slightly plus and keep invariant, slightly decrease, a little decrease, decrease, more decrease etc nine state classification..

\subsection{Simulation control model}

The controlled object of system is the stepper motor. The mathematical model can be expressed as the two order inertial link with the pure delay. Before the experiment, mathematical model of the stepper motor is obtained by using the least square method in system identification method. Generally, the motor control system is composed of two order inertia link and a pure lagging link.. According to the experimental data of the system identification, the model of the motor can be obtained as Eq. 1[5]:

$$
G(s)=\frac{k e^{-\tau s}}{s(T s+1)}
$$

Where $k$ is proportional coefficient, value is 2.93 ; $\tau$ is pure lag time, value is 0.06 , $T$ is time constant, value is 1.77 .

From the above analysis, the control effect of the nine point logic rule controller is largely determined by the error, error change and the choice of the nine kinds of control stepping motor's 
deflection angle. Therefore, how to determine these parameters and analyze the influence of the system dynamics and the steady state response is the key of the simulation study. The AFC logic control system simulation block diagram is shown in Fig. 2.

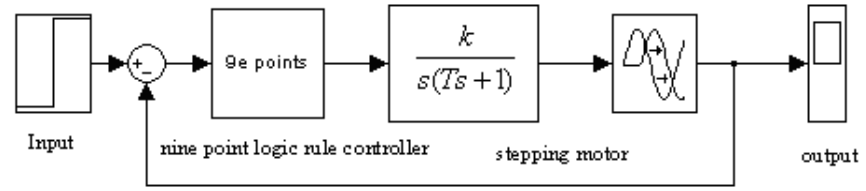

Fig 2 AFC logic control system simulation block diagram

\section{Simulation research of AFC logic control system}

Using the MATLAB software tools, the system offline in the SIMULINK environment for the role of logic rule controller simulation is studied. The simulation object is AFC logic control system. If the control accuracy is set as $0.005^{\circ}$, the error $e_{0}=0.005^{\circ}$ is set too. AFC logic control system simulation structure diagram is shown in Fig. 3 . In Fig.3, value of $\mathrm{K}$ indicates that the size of the control force and the gain parameter of the closed-loop system can be changed to change the quality parameters, so it can achieve the purpose of adjusting the dynamic and static characteristics by changing the gain parameters.

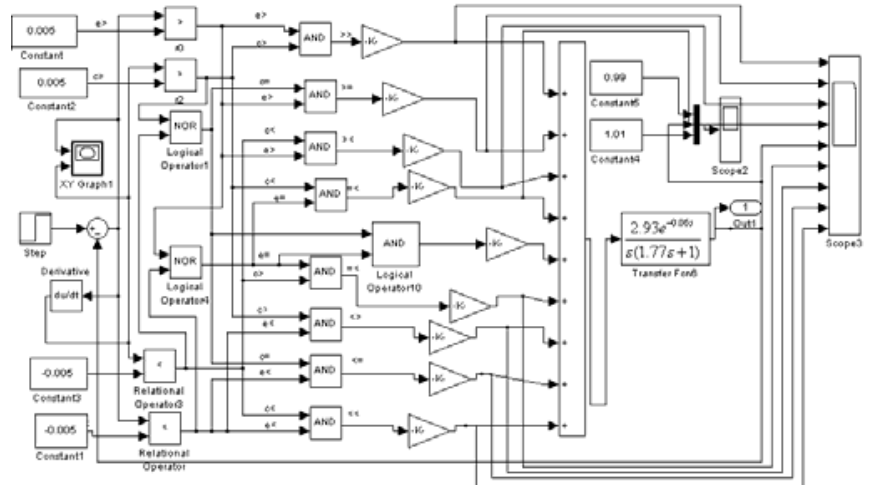

Fig. 3 AFC logic control system simulation structure diagram

Step signal is a common input signal in AFC control system, which is used in various control algorithms. To facilitate the study, set the target angle is 1 degree. The response curve of the AFC logic control system to a step signal is shown in Fig. 4.

The AFC logic control system is very important for the fast tracking of input signals in practical application. The dynamic tracking performance of the AFC logic control system is reflected by the tracking of the input signal. The amplitude of the sinusoidal signal is still set as 1 degree. The AFC logic control system under the action of nine point logic controller to track the sinusoidal signal is shown in Fig.5.

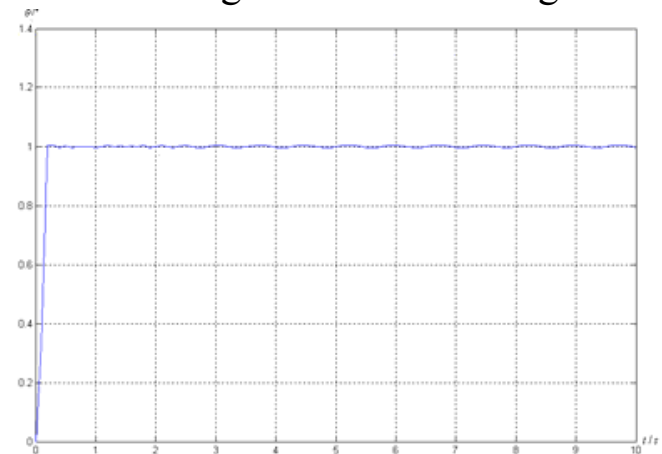

Fig.4 The response curve to step signal

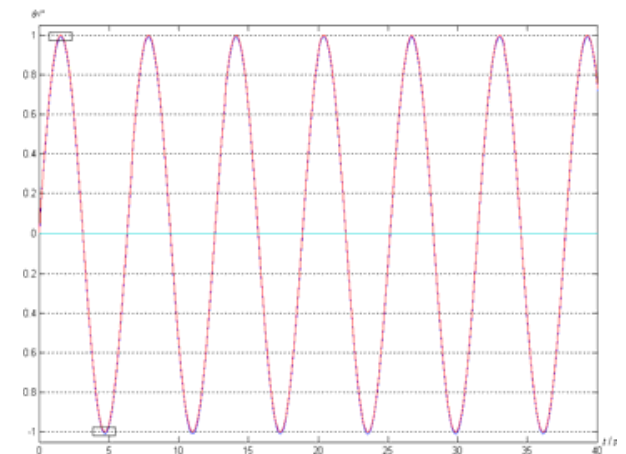

Fig.5 The effect curve to track the sinusoidal signal

The simulation results show that the delay time and the object model of the logic rule controller are not strict. In addition, by adjusting $K_{2+}$ to shorten the rise time, so that the system can quickly 
respond, and by adjusting $K_{4-}$, it can reduce or even eliminate the overshoot till meet the requirements of the system[6].

\section{Conclusion}

Nine point logic controller is used to control in the phase plane method. The control force is used in different regions. The parameters are easier to be adjusted, and the control results are better. After the simulation of AFC logic control system, the results show that this process is feasible and effective, and the control system has a simple structure. Adjustment is convenient, it is worth to be applied in AFS system.

\section{References}

[1] Zheng Weiliang. Development trend of automobile headlamp[J]. Automotive electronics, 2006, (10): $92-95$

[2] A. Peña-García, P. Peña, A. Espín, F. Azna. Impact of Adaptive Front-lighting Systems (AFS) on road safety: Evidences and open points[J]. Safety Science, Volume 50, Issue 4, April 2012, Pages 945-949

[3] Sun Xiaoming, Zhang Nanguan. Basic logic controller[C]. Chinese Society for artificial intelligence. The ninth annual meeting of the Chinese Association for artificial intelligence. Beijing: Beijing University of Posts and Telecommunications Press.2001, 188-189

[4] Sun Xiaoming, Zhang Nanlun. Nine point controller [C]. The fourth global intelligent control and Automation Conference. Shanghai: East China Science and Technology University Press, 2002.644-648

[5] Lin Guoyu, Wang Dong. A PID based on fuzzy AFS control method[J]. Micro computer Information, 2011, 27(10):1-3

[6]Sun Xiaoming, Zhou Rong, Zhang Nanguan et al. Study on the gain parameters of nine point controller[J]. Journal of Wuhan University of Technology, 2002, 24 (8): 80-82 